\title{
Is Someone Watching Me? - Consideration of Social Facilitation Effects in Human-Robot Interaction Experiments
}

\author{
Sarah Woods, Kerstin Dautenhahn, Christina Kaouri \\ School of Computer Science \\ University of Hertfordshire \\ College Lane, Hatfield, AL10 9AB, U.K. \\ \{s.n.woods, k.dautenhahn\}@herts.ac.uk
}

\begin{abstract}
This discussion paper considers the implications of the psychological phenomena of social facilitation effects for human-robot interaction (HRI) studies. Research studies in HRI have significantly increased over the past few years. Such studies investigate e.g. robot appearance and behaviour, and the responses of subjects to robots. However, the possible effects of the experimental context on results from human-robot interaction studies have attracted little attention. In this paper we provide an overview of robot trials with children and adults, as part of the Cogniron project. Observations from video footage are reported, with particular consideration for the influence of the social context and social facilitation effects, including task complexity, evaluation context and type of presence on outcomes of human-robot interaction studies. Lessons learnt and future design implications for human-robot interaction studies are provided.

Index Terms - human-robot interaction, social facilitation effects, design implications, adult and child trials, video footage.
\end{abstract}

\section{INTRODUCTION}

A relatively new area for robotics research is the design of robots that can engage with humans in socially interactive situations. Traditional service robots that are used e.g. to deliver hospital meals or provide security services often require only minimal human-robot interaction [1]. Different examples of robots designed to facilitate robot-human interaction include AIBO [2], Kismet [3], and Feelix [4]. Dario, Guglielmelli and Laschi [5] have provided a useful classification outlining the evolution of robotics. The evolution begins with basic robots within a structured environment such as within industrial automation and manufacturing, to personal robots designed e.g. for use by the disabled and elderly within the home.

Researchers are becoming increasingly interested in many different aspects of human-robot interaction including personality [6-9], speech [10], gestures [11], emotions [4, 12], posture, movement [13], and robot appearance [14-17]. However, the majority of HRI studies to date have adopted a robot-oriented approach by focusing on the robot's appearance, behaviour, perceptual abilities, control architecture, interactive skills, and cognitive abilities. One of the goals of our work in the Cogniron project (Cognitive Robot Companion) is to explore the user perspective of robot interaction, therefore adopting a human-centred approach. Investigating robot-human interaction from a human-centred perspective involves not only a consideration of the technological requirements of such a robot, but the study of psychological, social and cultural factors, which is a great challenge for HRI robotics research $[5,8,16,17]$.

In this discussion paper we will report on observations made during two different experimental robot trials conducted as part of the Cogniron project investigating different aspects of human-robot interaction. One trial involved groups of children and the other experiment involved individual adults. These different robotic experimental conditions, have highlighted the complex nature of human behaviour under different situations. Explanations for different interaction styles must take into account age, differences between the participants, and the distinct nature of the tasks that people were involved in. For example, the children's interactions with the robot were within a 'playful' context whereas in contrast, the individual adult trials were under the pretext of 'goal' oriented tasks. However, aside from these differences, we believe the mere presence of other people played a significant role. In this paper, we propose that psychological research findings into the effects of social facilitation could be insightful in explaining some of the human-robot interaction effects recorded. Findings about the mere presence of others in experimental situations could provide some useful guidelines to the wider robotics community for designing and implementing different experimental paradigms. It needs to be emphasised that our trials were not initially designed to study social facilitation effects.

The remainder of this paper is structured as follows. First, we will outline the nature of the different robot trials with the children and adult participants. We will then provide some examples of case studies from the child and adult robot trials, and some overall observations that were made. Psychological research findings into the effects of social facilitation will then be presented and the relevance of these findings to the observational data from the human-robot interaction trials will be considered. Lessons learnt from the experiments conducted and future design implications for robot studies involving human subjects will be proposed.

$$
\text { II. METHOD }
$$

The human-robot interaction trials

Group-Child interaction study using a play scenario:

In June 2004, 24 exploratory sessions involving 194 children aged 9-11 years (103 boys, 91 girls) were carried 
out at the University of Hertfordshire. The aim of the study was to consider non-verbal social interactions with regard to spatial distances between a robot and children. Research questions enquired about how the robot could attract children's attention, children's reactions to being the focus of the robot's attention, and how the children's activities were directed towards the robot as opposed to other children. A two by two design, with four experimental conditions was used, a moving/static pointer (selecting children) condition, and a moving/static camera (expressing "attention") condition.

Groups of 10 children participated in the trials and each session consisted of the robot playing two interactive games:

1) Rotation game: the robot revolved in the middle of a circle of children, stopped and selected a child by beeping twice and using different pointing conditions. During each round of the game (6 rounds) the selected child was removed from the circle, and the rest of the group moved $0.5 \mathrm{~m}$ closer to the robot.

2) Wander game: The same procedure was used but at each round the remaining children did not move towards the centre and the robot. The robot wandered randomly around the circle of children and then selected a child by facing the child from a distance of $800 \mathrm{~mm}$ and making 2 beeps.

The trials involved PeopleBot ${ }^{\mathrm{TM}}$ robots that were fitted with a lifting arm, which acted as a pointer (Fig. 1). The robot had a small basket holding presents that were presented at each round of the game. Each of the trials was videotaped to allow researchers to analyse more closely the movements, postures and behaviours of the children.

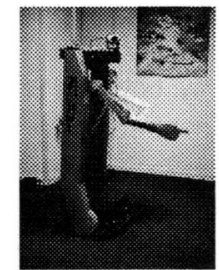

Fig. 1 PeopleBot ${ }^{\mathrm{TM}}$ robot with the lifting arm used in the children's robot trials

Questionnaires were administered before and after the children took part in the rotation and wander game (Figs. 2 \& 3).

Individual-Adult interaction study using a simulated living room scenario:

This study explored how adults interacted with a single robot in a simulated living room scenario (Fig. 4). Twentyeight adults recruited from the University of Hertfordshire participated in the robot trials (using a PeopleBot ${ }^{\mathrm{TM}}$ robot), which concentrated on a human-centred perspective in terms of how the robot's appearance and possible personality attributes were perceived. Levels of comfort with the robot, and the idea of possible robot companions for the home were also considered.

This study was different from the child robot trials, as a more "serious" task-oriented approach was taken. Adults took part in two different tasks, a negotiated space task involving moving within the same restricted working area as the robot, and an assistance task where the robot interacted with the adult and assisted them with fetching pens. Each adult was exposed to the tasks twice, on one occasion the robot behaved in a 'socially ignorant' manner and in the other instance a 'socially interactive' manner. The order of presentation was counter-balanced and the behaviour styles of the robots were predefined. During the tasks, subjects were asked to solve problems that were meant to be simple and not involve a high cognitive load. Our intention was to provide contexts where the subjects were kept 'busy' (comparable to a person involved in different activities in her home, e.g. reading a book or cooking), but still provide enough opportunity to observe human-robot interactions.

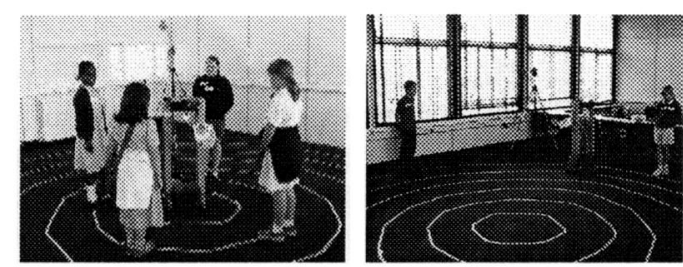

Fig. 2 \& 3 Children playing 'rotation' game and 'wander' game

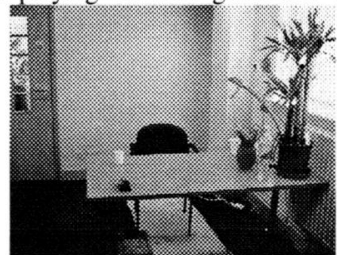

Fig. 4 The simulated living room for the adult interaction trials

Research questions enquired about different situational contexts (cf. Fig. 5), e.g. we investigated which robot behaviour style subjects preferred when the human subject and robot were carrying out different tasks but within the same limited work area.

Adults entered the simulated living room and were introduced to the robot (co-habituation), before completing some initial questionnaires about adult demographics and personality styles. Comfort and social distance tests were conducted between the adult and robot to determine adult comfort levels with the robot. The negotiated space task was carried out with the socially ignorant or socially interactive behaviour of the robot randomised. The assistance task followed with the same robot behaviours. A series of other questionnaires were completed by the adults before they interacted in the same robot tasks again but with the alternate robot social behaviour. At the end of the trials, subjects completed a further questionnaire about their views toward a future robot companion. Results from the questionnaires are presented elsewhere. All trials were videotaped.

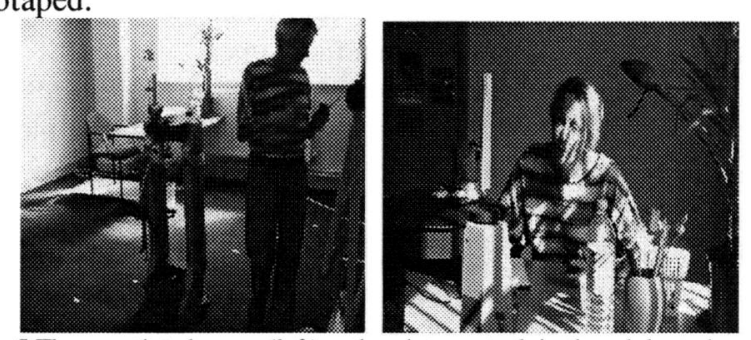

Fig. 5 The negotiated space (left) and assistance task in the adult study. The role of the experimenter

Important differences between the role of the experimenter during the child and adult robot interaction trials should be highlighted. During the children's robot 
trials, the experimenter dealt with the overall running and management of the game. A guidance role was assumed throughout the trials. They provided instructions to the children about the trials and remained at the periphery of the game to ensure everything ran smoothly. In contrast, the role of the experimenter in the adult trials was more structured. A set of fully standardised instructions was used for each subject. Once the subjects were provided with instructions, the experimenter did not have any eye contact or initiate interaction with them unless directly, verbally, addressed with a question. To maintain this, the experimenter appeared to be reading a large broadsheet newspaper to avoid giving away behavioural cues to the subject.

\section{TRIAL OBSERVATIONS}

In retrospect, we are aware that the comparison of the group studies with children and individual studies with adults are not strictly comparable due to the different contexts etc. As noted above, the trials were not specifically designed for the study of social facilitation effects. However, the already existing results from these HRI studies served as a starting point for our discussions of social facilitation effects.

\section{Observations from group child-robot interaction trials}

Different interaction styles were seen depending on the group set-up, and whether a teacher was present or not in addition to the experimenter. A few selected cases are described below based on observations from the video data.

\section{Child-group robot trials in the presence of an experimenter \& teacher - Rotation Game:}

When the children were introduced to the robot, they initially sat at quite a distance from the robot, but then two girls moved closer to the centre of the circle towards to robot. The rest of the group then followed. One of the boys then reached out to touch the robot's hand. When the rotation game trial began, all the children tried to have eye contact with the robot when it was pointing with the camera towards them. All the children stood very still with their arms by their sides when the robot was moving around. They looked to each other frequently when the robot initially started moving, and they all giggled when the first boy was chosen by the robot. When the robot started moving again, they became quiet. One of the boys was quite distractible during the game, and put his hands on his hips and seemed to get fed-up waiting for the robot to select a child. The girls tended to smile among each other more than the boys. The closer the children were to the robot, the more they looked at each other, maybe for reassurance of what the robot was doing. When the robot was facing a particular child (camera at the front), the children tended to smile at the robot.

\section{Child-group robot trials in the presence of an experimenter} only - Rotation Game:

When this group of children were introduced to the robot, they all sat right in front of the robot (camera and arm side) and started chatting loudly amongst themselves. They seemed to be fascinated with the robot's hand and also wanted to grab the toy 'bugs' from the basket attached to the robot. Some children in the group reached out to touch the robot's hand. This group were much noisier and verbal compared to the group where the teacher was present. In particular, one boy who appeared to act as the group leader influenced the rest of the group. He kept clapping his hands and shouting out very loudly "me, me pick me". This seemed to open up the opportunity for the rest of the group to start saying things to the robot, among "hello, you stupid robot, why won't you pick me?" and "hello, you silly thing". The boy that initiated this dialogue with the robot then started to dance in the hope of getting the robot's attention. When the boy selected by the robot in the first round of the game left the circle he shouted out "I'm dead now". When the next round of the game began the same boy started dancing again and said "If you dance, the robot might pick you". The girls in the group did not seem to pay much attention to this, they stood very still did not verbalise much. The boy who was dancing then moved off the line where the children were supposed to stand, moved toward the robot and shouted out "it wouldn't pick me because of my dance". The boy then flicked the robot's camera. Another boy then started copying the 'dancing' boy and shouted out "oh come on, hurry up and decide".

Child-group robot trials in the presence of an experimenter and teacher - Wander Game:

One of the boys in the group waved at the robot to try and make it start the game. At this point there was a lot of giggling among the group and all the children started looking at each other. There was a lot of eye gaze and attention towards the robot by the children when it was roaming around the room. Some of the children started to wave at the robot to try and get its attention and make it wander towards them. It seemed that two of the boys became somewhat bored with this game and started to look around the room and behind them. There was some chatting among the group. For example, when the robot was slightly delayed in moving, they said 'he's asleep'. When the robot approached one of the girls from the side, it seemed that she was not expecting this and jumped slightly. She then moved the position of her body to face the camera and arm side of the robot.

\section{Child-group robot trials in the presence of an experimenter} only - Wander Game:

During the wander game the children were again much louder and more verbal compared to the group where both the teacher and experimenter were present. This group also attempted to gain the robot's attention with different body movements (e.g. waving, dancing) more than the group with the teacher present. At the beginning of this game, one of the children, when it saw the robot approaching another child said "I've got chocolate, come to me". This caused a lot of giggling among the group. Another child began to get frustrated with the robot and shouted out, "Oh come on, it hates me, stupid robot". Another girl when the robot started to approach her said "he likes me". Another child then caught onto the notion that it might influence the robot if it tried to entice it and shouted out "I've got a million pounds robot, come to me, come to me". Children in this group also 
attempted to name the robot "I name it, Maple school property robot, property of Maple school" and another names it "robot chick". When the robot started to move towards one of the girls that named it, she said "see it answers to its name, I told you that it did".

\section{Observations from individual adult-robot interaction styles}

Observations from the video data of individual adult robot trials were completely different from the child robot trials. It was clear from the video footage that the adults were influenced by the presence of the experimenter (e.g. looking towards her for social referencing). The style of adult interactions observed, irrelevant of the robot's behaviour (socially ignorant, socially interactive) ranged from being confident and chatty with the robot to extremely nervous and non-communicative with the robot. Below, we illustrate in more detail the behaviour of five subjects within this spectrum of behavioural responses:

Adult one (female) displayed confident behaviour towards the robot. She chatted with the robot and responded immediately with "hello" when the robot said hello to her. When the subject needed more pens during the assistance task she said "thank you very much" to the robot when they were given to her. This subject had lots of eye contact with the robot and had a relaxed body posture. She also smiled frequently at the robot and said "very nice" and "thank you robot" on a frequent basis. This subject had good task concentration and was able to distribute her attention between the task and robot appropriately (i.e. she wasn't constantly checking to see what the robot was doing, or where the robot was at a cost of completing the task). However, the subject attempted to see how far the robot's abilities extended to and tested to see if it was preprogrammed perhaps, as she initiated conversation with the robot and said "Can you get me a highlighter pen?" The robot then responded with its pre-scripted response "I see that you need some more pens". This subject did not appear to make reference to the experimenter by gazing toward her or asking any questions throughout the trial.

Adult two (male) on the other hand did not engage in any conversation with the robot but did begin to laugh at the robot after a while and smiled occasionally at the robot. This subject appeared to be apprehensive in the presence of the robot to begin with, and was a bit jumpy whilst doing the assistance task as he kept looking up to check on the status of the robot. This subject also checked on the experimenter from time to time, but did not receive any feedback. No questions were directly asked to the experimenter.

Adult three (female) came across as a bit puzzled at the beginning of the assistance task and started to stroke her face gently. She had good eye contact with the robot but at times displayed an unsure smile at the robot. This subject had little conversation with the robot other than saying "thank you" when the robot bought her the pens. She referred to the experimenter during the trial, although not verbally and no feedback was provided.

Participant four (male) appeared to be shocked and puzzled when the robot first spoke to him during the assistance task. He had a very inquisitive, unsure look on his face for quite a while. He hesitated during the task and kept looking up at the robot to see what it was doing. $\mathrm{He}$ frequently frowned and raised his eyebrows when the robot made a new noise. He was totally puzzled by the robot's behaviour when the pens were dropped off at the desk. This subject did not have as much eye contact with the robot compared to others and seemed happier ignoring the robot and not paying it any attention. The subject laughed when the robot made a mistake and knocked over the flowers on the desk when dropping off the pens for the task. He raised his cup as a signal of thanks at the end of the trial, but did not attempt to initiate conversation with the robot. When he left the room at the end of the trial, he raised his book, seemingly as a sign of goodbye.

Subject five (female) was the most apprehensive and nervous in the presence of the robot. She jumped when the robot spoke to her and seemed really unhappy and uneasy throughout the trial. She kept making reference to the robot's camera and appeared to be nervous and annoyed when the robot stayed at the desk and didn't move further away. Even though the robot was near to her and had been for a few minutes, this subject jumped with surprise and had a shocked expression on her face when the robot said "I notice that you need more pens". She responded with a nervous smile and continued by biting her lip anxiously.

Based on the above observational data, a number of themes emerged from the trials:

- Overall, children were much more confident during the group interactions than adults were during the individual interactions.

- Conversation was used more frequently during the group child interactions toward the robot compared to the adult individual trials.

- Children did not seem to make reference to the experimenter during the group trials, however, adults frequently checked on the status of the experimenter in the individual trials.

- More apprehension and puzzlement was exhibited towards the robot in the adult individual trials compared to the group child robot interactions.

- Discrete body language and non-verbal cues were more evident in the adult trials compared to the child trials.

- Children frequently went right up to the robot to inspect it, even when they were not instructed to, and started closely examining the camera and the robot's arm. None of the adults did this with the robot's camera.

- Children assigned a name to the robot, and a gender on some occasions, and tried to use tactics to attract the robot and get the robot to approach them. No adults exhibited this kind of behaviour.

- The presence of a teacher during the child robot trials seemed to have a large effect on the amount of conversation the children exhibited and body movements toward the robot.

- Children in the group situations copied and imitated each others' actions frequently in an attempt to get the robots attention (e.g. the two boys dancing). Copying of actions of other people did not occur in the adult trials. 


\section{SOCIAL FACILITATION EFFECTS}

In this section, we provide possible explanations of our observations in relation to social facilitation effects as discussed in the literature. Lessons learnt and implications for HRI studies are presented.

The robot trials described above involved different group configurations ranging from individual adult trials with just the experimenter present, to group child interactions with the experimenter, and sometimes a teacher present. An exploration of psychological research into social facilitation effects in groups could be useful in explaining the different robot-human interactions and the future design of robot experiments paying close attention to the environmental context and group structure. Few studies within robotics research have considered the phenomena of social facilitation. One exception is the study carried out by Bartneck [18] which found that participants gained higher scores in a robot condition compared to a screen condition. Results were explained in terms of social facilitation effects and that the robot character appeared to have stronger social facilitation effect than the screen character resulting in participants putting more effort into the negotiation. In the following sections, social facilitation effects will be explained from a psychological perspective with examples from our human-robot interaction trials. These examples are hoped to highlight the importance of considering social facilitation contextual effects for human-robot interaction studies.

\section{Evidence from Psychology (1)}

Social facilitation is one of the oldest social psychology theories in the history of the field of psychology [19]. The theory focuses on changes that occur when individuals perform tasks alone or in the presence of others, and has been defined as the extent to which a given piece of an individual's behaviour is influenced and improved as a result of the real, imagined or implied presence of others [20]. However, social facilitation effects are not straight forward as research is increasingly reporting that the relationship between social presence and individual performance is influenced by: Task Complexity, Evaluation Context and Type of Presence.

In fact, under some circumstances, findings have revealed that performance is inhibited rather than facilitated [19]. Currently, there is still no single theory that can effectively and parsimoniously explain the phenomenon of social facilitation [19]. Below, we provide an overview and description of some of the earliest studies into social facilitation, followed by a summary and critique of different psychological explanations for social facilitation effects.

The social facilitation paradigm is rich in history and dates back to the original experiments on pacing and competition, carried out by Triplett in 1898 [21]. Triplett noted that bicycle racers turned in faster times when they were racing with each other, than when they raced alone. The fastest times were for those cyclists who competed against each other and the slowest times were observed for those who raced against the clock with no pacesetter. In another experiment, Triplett found that most children reeled fishing reel faster when they were reeling alongside another child. Triplett proposed that the presence of a co-actor stimulated a competitive instinct that motivated the individual to reel faster.

However, the experiments conducted by Triplett all contained elements of competition. Allport [22] coined the term 'social facilitation' and attempted to control for competition effects by carrying out experiments using two kinds of mental tasks, word associations and generation of arguments to a written passage. Results revealed that people in group situations made a higher number of associations and generated a larger number of arguments. However, the quality of the arguments generated was better when subjects were alone. This led Allport to suggest that task performance is affected differentially by social presence. Travis (1925) [in 23] proposed 'audience effects' after he observed clear improvements in performance on the pursuitrotor task, when subjects were confronted with an audience. These findings were supported by Dashiell [24] who reported enhanced performance for simple tasks when an audience was present. However, some researchers found negative audience effects. For example, Pessin (1935) (in [23]) reported that students made more errors in the 'audience' condition when the task was to recall nonsense syllables compared to the 'alone' condition.

Possible Implications for HRI studies based on the Cogniron robot trials $(1)$

The human-robot interaction trials carried out as part of the Cogniron project emphasised to both the child and adult groups that their performance was not being judged and that there were no correct or incorrect interaction styles. However, based on the video observations, it was clear that some of the children were competing with each other to try and get the robot's attention and get it to select them. In the adult single trials, none of the adults actively tried to get the robot to approach them or instruct it to do something for them. However, indirect elements of competition were also evident from the adult trials. Even though, the activities chosen for the trials (e.g. copying words onto a board and highlighting words) were designed to be non-competitive with no directly observable achievement goals, the adults still behaved as if they were under exam conditions, and took the tasks extremely seriously. The majority of the adults expressed little fun or enjoyment during the tasks, instead expressions of hard concentration were observed, despite the fact that the experimenter emphasised the nonperformance based nature of the trials. If a more relaxing, leisurely athmosphere is desirable, then the experimental setup needs to be revised. To assist in obtaining these conditions, a longer habituation time between the adult and robot may be necessary. Alternatively, studies carried out in the field rather than the laboratory could eliminate some of the issues surrounding competition and task performance [25]. For example, robot trials in subjects' homes would be advantageous because although we tried to furnish the conference room at the University to resemble a simulated living room as closely as possible, all the subjects knew that it was not real or natural. Ultimately, adults' focus on task performance could have masked some important findings about their perceptions of the robot, as they may not have observed enough of the robot's behaviour and appearance to form concrete perceptions and attitudes, but more the 
content of the tasks and their individual performance on it. Future instructions could clearly state that the trials focus on the robot's behaviour rather than the subjects' performance, and that subjects should pay close attention to the appearance and behaviour of the robot interactions.

Evidence from Psychology (2)

Zajonc noted discrepancies in research findings concerning audience and alone conditions, and concluded that well-learned responses are facilitated by the presence of spectators, while the acquisition of new responses is impaired. Therefore, performance is facilitated and learning is impaired by spectators [23]. Zajonc drew a distinction between dominant and non-dominant responses, noting that some behaviours are easier to learn and perform than others. If a task is easy for the person, then the dominant response will be the correct one (i.e. most likely) and therefore the audience/co-actor helps to elicit this. However, sometimes the dominant response is the incorrect one(s) (i.e. the most likely again), but the audience still assists in the elicitation of this response.

Support was generated for the mere presence effect in an experiment carried out by Guerin [26] where participants performed a rotory-pursuit task in one of three conditions: alone, with a simple distraction (a large mirror placed alongside the participants), and in the presence of a (passive) confederate. The confederates did not watch the participants and did not know what the experiment was about. Evaluation effects should not have played a role because the participant could not complete the task and look at the confederate at the same time. Results revealed that subjects performed better when the confederate was present compared to when they were alone or had the distraction of the mirror. Platonia and Moran [27] reported similar results.

Attention has concentrated on the 'alone' condition in some studies, as it has been suggested that this is a poor control for social facilitation experiments. For example Griffin and Kent [28] demonstrated that by simply giving participants a task to perform within laboratory conditions leads them to assume that their performance is being monitored, even without the presence of an audience. Griffin [29] carried out a study with psychology undergraduates involving four conditions: in two conditions an identical card-sorting task was performed either with or without a stopwatch, in the third condition, the card-sorting task was performed in front of an audience of two (one man, one woman), and in the fourth condition, participants waited alone, without a task. Results from questionnaires revealed that participants inferred monitoring in both of the task conditions. Participants who waited alone without a task did not infer monitoring.

Possible Implications for HRI studies based on the Cogniron robot trials (2)

In our robot trials, subjects were told that the experimenter was not part of the task and was not there to monitor individual performance. Although we did not assess the extent in which subjects felt monitored, the video cameras that we used in the adult trials were pointed out at the outset of the trials. Subjects were told that the sole purpose of the cameras was to monitor the robot's behaviour. The extent to which participants felt monitored could be an important factor for future studies to consider as it may influence robot-human interaction styles. This is a difficult situation to overcome for human-robot interaction studies as even without the direct presence of an experimenter, simply giving subjects a task to perform appears likely to elicit some feelings of performance being monitored. The experimental context however could assist in reducing feelings of being monitored. It was clear from the observations of the robot trials that the children in group situations felt much more comfortable and less monitored than the adults did in the alone condition. The nature of the tasks could have had an impact on this also, as the child trials had a much more game-like, relaxed content compared to the adult robot trials. It would be interesting in future adult trials to explore the impact of group size (i.e. pairs, groups of 4) on perceptions of the robot.

Evidence from Psychology (3)

Other studies have considered the effects of group versus alone conditions in relation to impression formation [30]. Thomas et al. carried out an experiment to test whether Zajonc's drive theory of social facilitation also predicted how the mere presence of others influenced social judgement ratings. Participants were exposed to different conditions involving a positive and negative experimenter behaviour condition, and a mere presence condition, group or alone. Results revealed that people formed more extreme impressions of a target when they made judgements in the mere presence of others. This was found for both positive and negative behavioural effects and is consistent with the drive theory of social facilitation.

Possible Implications for HRI studies based on the Cogniron robot trials (3)

Little attention has been paid to the effects of an experimenter in human-robot interaction studies, and may imply confounding variables. For example, in the current robot experiments, it could be the case that the children formed more extreme positive and negative opinions toward the robot under the group condition compared to the adults who performed the interactions alone. Children in groups were able to discuss between themselves what they felt towards the robot and verbalised frequently about 'the robot being stupid because it didn't approach them' or 'that the robot was clever because it came to them'. Discussing opinions within a group situation is an important aspect of impression formation. However, the adults did not have this opportunity and were unable to confirm or disconfirm their attitudes and perceptions towards the robot with anyone, which could account for the frequent appearances of puzzlement on subjects' faces. The same experimenter was present at both the child and adult robot trials, and never exhibited negative behaviours, so this was unlikely to have had an impact on the robot trial results.

A further important consideration is whether the presence of others facilitates or inhibits emotional expression. For example, in our human-robot interaction trials, we are interested in capturing the emotional responses and reactions that participants displayed in response to the different robot scenarios. However, it is possible that the video footage of emotional responses was affected by the group and individual conditions, meaning that direct 
comparisons should not be made. Ross et al. [31] carried out a study to examine the potential effects of group structure on emotional responses. Participants viewed emotionally loaded slides with either friends, strangers or alone. Results revealed that strangers had overall inhibitory effects on communication accuracy, whereas friends had facilitative effects on slides showing positive emotions and inhibitory effect on emotionally negative slides. Results for the alone condition were somewhere in between the stranger and friend conditions. These effects could have been evident during our robot trials. For example, the children were in the presence of their classmates and friends, which could have facilitated the expression of different emotions during the robot trials. In contrast, an inhibitory effect on emotion expression may have occurred with the adult trials as they might have tried to conceal their emotions as they felt more self conscious not having anyone to confirm or discuss their feelings with.

\section{Theoretical explanations for social facilitation affects}

Guerin [32] classified social facilitation effects according to three theoretical perspectives, drive theories, social comparison theories, and cognitive process theories. Drive theories were inspired by Zajonc [23] and were based on the Hull-Spence drive theory, which posited that in the presence of others, individual drive levels are elevated. When arousal arises from a difficult or unfamiliar task, this results in stress and consequently poor performance. This extra arousal results in taking people past the optimum arousal level and results in the dominant response being elicited whether it is easy, or new and difficult. Zajonc was challenged on his 'mere presence' explanation, but later asserted that social facilitation effects still emerged even when the situation and behaviour of others were controlled for. For example, factors such as evaluation apprehension could influence individual reactions to the presence of others, but Zajonc still claimed that mere presence was necessary and sufficient for social facilitation. Cottrell [33] was one of the proponents that challenged Zajonc's drive theory, and stated that mere presence was not enough to elevate drive levels and would not necessarily cause social facilitation effects. Cottrell proposed the evaluation apprehension effects hypothesis stating that increases in drive levels were a result of individuals being concerned about how others would evaluate them. Cottrell also stated that prior evaluation experiences caused people to develop a drive reaction - a learned drive.

An example of a social comparison theory is the Selfpresentation explanation. Self-presentation assumes that social facilitation effects are focused on impression formation and the well-established finding that people are motivated to please those that are observing them, sometimes referred to as social desirability effects. Baumeister [in 19] for example suggested that the presence of someone considered to be evaluative would trigger more drive than the presence of someone not evaluating performance.

Cognitive processes as an explanation for social facilitation effects often emphasise distraction (in [19]). For example, attention conflict (Baron 1986, in [19]) can produce drive like effects on performance that can facilitate simple tasks and impair complex ones.

Future directions for the design of social facilitation studies and the implications for human-robot interaction research

Aiello and Douthitt [19] highlighted some of the issues that future research studies into social facilitation research should consider. Firstly, studies should consider the nature of the group composition and levels of familiarity. For example, effects might be different for friends, family members, coaches, teachers, supervisors etc. Most studies to date have been carried out with groups of participants who are assumed to be strangers. However, the child robotinteraction studies in the current human-robot interaction trials were carried out with groups of children from the same class, who were highly familiar and possibly friends with each other, and in some cases with the teacher present. In comparison, the adult robot-interaction trials were conducted with individual adults and just the experimenter who was assumed to be a stranger in most instances. The effects of group structure and familiarity could therefore have had a considerable impact on the results of subjects' perceptions toward the robot. Second, studies should explore the role of time and how long the predicted effects of social facilitation are expected to last. The practical implications for social facilitation effects would be different if they lasted over days or weeks, compared to a few hours. This is an important point to take into account for the robot trials as habituation effects with the task set-up and robot could greatly influence results. Tasks employed to study social facilitation effects have relied on the quantity and quality domains and have not taken into account contextual performance issues. Finally, measures used to explain social facilitation effects should be improved and should not rely too heavily on self-report assessments or through inferences from manipulating experimental conditions (e.g. condition where people are told they are being evaluated or not evaluated). Although, our human-robot interaction trials were not designed for measuring social facilitation effects, the importance of considering the effects of monitoring, group formation, social context, task complexity and the methods used to assess subjects perceptions towards robots should be carefully planned and piloted.

\section{CONCLUSIONS}

To summarise, we have presented observations from video footage data of human-robot interaction trials, and discussed these in light of psychological findings related to social facilitation effects. The design of research studies into human-robot interaction is growing. However, the possible effects of the social context where experiments take place is usually not addressed, although awareness of these effects is beginning to emerge. For example, a longitudinal case study trial using the service robot Cero emphasised the importance of considering the situational context when analysing and interpreting results and conclusions [25]. It can be expected that the social context of any HRI study will impact the results. This context will vary considerably depending on the scenario used, the type of robot, and desired purpose for the robot. For example in the case of 'toy' robots such as AIBO 
[2], and Kismet [3], where the only purpose of the robot is to engage people in interaction, the social context of humanrobot interaction is likely to be very different compared to adult interactions in a more 'serious' task context involving service robots (e.g. $[10,25,34,35])$. Also, the social context of a robot that serves as a museum guide or is shown at exhibitions is likely to impact how people will behave in the robot's presence. An observation of distinct differences between our child and adult robot trials under different social contexts resulted in a discussion of the relationship between social presence and individual performance in relation to task complexity, evaluation contexts and type of presence. The importance of acknowledging these social effects in future studies is emphasised, as ignoring these influences could significantly impact on findings, and ultimately the design implications for future robot companions. While the examples of human-robot interaction discussed in this paper were not specifically designed for the study of social facilitation effects, future work can take into account the issues that we raised.

\section{ACKNOWLEDGMENTS}

The work described in this paper was conducted within the EU Integrated Project COGNIRON ("The Cognitive Robot Companion") and was funded by the European Commission Division FP6-IST Future \& Emerging Technologies under Contract FP6-002020. Many thanks to Mick Walters, Kheng Lee Koay, Christina Kaouri, René te Boekhorst, Chrystopher Nehaniv, Iain Werry, \& David Lee for their help in designing, implementing \& carrying out the trials.

\section{REFERENCES}

[1] D. Wilkes et al., "Toward socially intelligent service robots," Applied Artificial Intelligence Journal, vol. 12, pp. 729-766, 1997.

[2] Sony, "Sony Entertainment Robot Europe http://www.aiboeurope.com/," 2004

[3] C. L. Breazeal, Designing sociable robots. Massachusetts: The MIT Press, 2002.

[4] L. Cañamero, "Playing the emotion game with Feelix: What can a LEGO robot tell us about emotion?" in Socially intelligent agents: Creating relationships with computers and robots, K. Dautenhahn, A. Bond, L. Canamero, and B. Edmonds, Eds. Massachusetts, USA: Kluwer Academic Publishers, 2002, pp. 69-76.

[5] P. Dario, E. Guglieimelli, and C. Laschi, "Humanoids and personal robots: Design and experiments," Journal of Robotic Systems, vol. 18 , pp. 673-690, 2001.

[6] D. C. Dryer, "Getting personal with computers: How to design personalities for agents," Applied Artificial Intelligence, vol. 13, pp. 273-295, 1999

[7] K. Dautenhahn, "Design spaces and niche spaces of believable social robots," Proc. IEEE Ro-man, Int. Workshop on Robots and Human Interactive Communication; RoMan, Berlin, Germany, 2002.

[8] S. Woods, K. Dautenhahn, and J. Schulz, "The design space of robots: Investigating children's views," Proc. IEEE Ro-Man, Kurashiki, Japan, pp. 47-52, 2004.

[9] K. Severinson-Eklundh, A. Green, and H. Hüttenrauch, "Social and collaborative aspects of interaction with a service robot," Robotics and Autonomous Systems, vol. 42, pp. 223-234, 2003.

[10] B. A. Maxwell et al., "Alfred: The robot waiter who remembers you." Proc. $8^{\text {th }}$ AAAI Workshop, Texas, USA, 2000.

[11] M. Scheeff et al., "Experiences with Sparky, a social robot," in Socially intelligent agents: Creating relationships with computers and robots, K. Dautenhahn, A. Bond, L. Cañamero, and B. Edmonds, Eds. Massachusetts, USA: Kluwer Academic Publishers, 2002, pp. 173-180.

[12] R. C. Arkin, Behaviour based robotics. Massachusetts: The MIT Press, 1998.
[13] M. Montemerlo et al. "Experiences with a mobile robotic guide for the elderly," Proc. AAAI National Conference on Artificial Intelligence, Edmonton, Alberta, Canada, 2002.

[14] T. Fong, I. Nourbakhsh, and K. Dautenhahn, "A survey of socially interactive robots," Robotics and Autonomous Systems, vol. 42, pp. 143-166, 2003.

[15] B. R. Duffy, "Anthropomorphism and the social robot," Robotics and Autonomous Systems, vol. 42, pp. 177-190, 2003.

[16] C. DiSalvo et al., "All robots are not created equal: The design and perception of humanoid robot heads," Proc. DIS2002, London, 2002.

[17] J. Goetz, S. Kiesler, and A. Powers, "Matching robot appearance and behaviour to tasks to improve human-robot cooperation," Proc, IEEE Ro-man, Milbrae, CA, 2003.

[18] C. Bartneck, "Interacting with an embodied emotional character," presented at DPPI, Pittburgh, Pennsylvania, USA, 2003.

[19] J. R. Aiello and E. A. Douthitt, "Social facilitation from Triplett to electronic performance monitoring," Group Dynamics, vol. 5, pp. 163$180,2001$.

[20] D. Forsyth, Group Dynamics. New York: Brooks/Cole-Wadsworth, 1999.

[21] N. Triplett, "The dynamogenic factors in pacemaking and competition," American Journal of Psychology, vol. 9, pp. 507-533, 1898

[22] F. H. Allport, "The influence of the group upon association and thought," Journal of Experimental Psychology, vol. 3, pp. 159-182, 1920.

[23] R. B. Zajonc, "Social faciliation: A solution is suggested for an old unresolved social psychological problem," Science, vol. 149, pp. 269$274,1965$.

[24] J. F. Dashiell, "Experimental studies of the influence of social situations on the behaviour of individual human adults," in Handbook of social psychology, C. A. Murchison, Ed. Worcester MA: Clark University Press, 1935, pp. 1097-1158.

[25] H. Hüttenrauch and K. Severinson-Eklundh, "Fetch-and-carry with CERO: Observations from a long-term user study with a service robot," Proc. IEEE Ro-man 2002, Int. Workshop on Robot \& Human Interactive Communication, 2002

[26] B. Guerin, "The effects of mere presence on a motor task," The Journal of Social Psychology, vol. 126, pp. 399-401, 1986.

[27] J. Platonia and G. Moran, "Social facilitation as a function of the mere presence of others," The Journal of Social Psychology, vol. 14, pp. 190$197,2001$.

[28] M. Griffin and M. Kent, "The phenomenology of the alone condition. The role of aloneness in social facilitiation," The Journal of Social Psychology, vol. 138, pp. 667-669, 1998.

[29] M. Griffin, "The phenomenology of the alone condition: More evidence for the role of aloneness in social facilitation," The Journal of Psychology, vol. 135, pp. 125-127, 2001.

[30] S. L. Thomas et al., "Social facilitation and impression formation," Basic and Applied Social Psychology, vol. 24, pp. 67-70, 2002.

[31] B. Ross et al., "Social facilitation and inhibition of emotional expression and communication," Journal of Personality and Social Psychology, vol. 63, pp. 962-968, 1992.

[32] B. Guerin, Social Facilitation. Cambridge, England: Cambridge University Press, 1993

[33] N. B. Cottrell, "Social facilitation," in Experimental social psychology, C. G. McClintock, Ed. New York: Holt, 1972, pp. 185-236.

[34] S. Thrun, et al., "Probablistic algorithms \& the interactive museum tour-guide robot Minerva," Journal of Robotics Research, vol. 19, pp. $972-1000,2000$

[35] H. Hüttenrauch \& K. Severinson-Eklundh, "To help or not to help a service robot," Proc. IEEE Ro-man 2003, Int. Workshop on Robot \& Human Interactive Communication, Millbrae, CA, USA, 2003. 